\title{
Ultrashort laser pulses: a comparison between a mathematical model and experimental data
}

Roberto Ortega-Martinez, Carlos Roman-Moreno, Antonio Rodriguez-Rosales, Cesar Juarez-Melchor

Roberto Ortega-Martinez, Carlos J. Roman-Moreno, Antonio A. RodriguezRosales, Cesar A. Juarez-Melchor, "Ultrashort laser pulses: a comparison between a mathematical model and experimental data," Proc. SPIE 3831, Sixth International Conference on Education and Training in Optics and Photonics, (16 June 2000); doi: 10.1117/12.388697 


\title{
Ultrashort laser pulses: A comparison between a mathematical model and experimental data
}

\author{
Roberto Ortega-Martínez, Carlos J. Román-Moreno, \\ Antonio A. Rodríguez-Rosales and César A. Juárez-Melchor \\ Laboratorio de Pulsos Ultracortos, Centro de Instrumentos, UNAM. \\ Cd. Universitaria, A.P. 70-186, México, D.F., 04510, MEXICO.
}

\begin{abstract}
The spectral properties of almost-Gaussian functions are considered and applied to the characterization to the second-order approximation in the expansion of the coefficients of almost-perfect optical pulses. Specifically, adding small amounts of odd-order Hermite-Gaussians to a Gaussian induces a second-order increase in the time-bandwidth product, while the increase in the time-bandwidth product from adding even-order Hermite-Gaussian is higher-order and hence smaller. We indicate the class of small perturbations of Gaussian functions which change neither the temporal profile of the intensity nor the intensity of the spectral profile. We compare the almost-Gaussian functions with femtosecond temporal width pulses data given by a Ti:Sapphire laser.
\end{abstract}

Keywords: Ultrashort pulses, lasers, optical mathematics.

\section{INTRODUCTION}

The last few years have brought about dramatic advances in the physics and technology of ultrashort-pulse generation over a wide range of the electromagnetic spectrum. The techniques and technology of ultrafast sciences provide a unique method for investigating physical phenomena throughout the physical and biological sciences. "Ultrafast" is a term typically used to describe processes which occur on a picosecond $\left(10^{-12} s\right)$ or faster time scale. The primary sources of coherent short-pulse radiation have been passively mode-locked lasers, which emit continuous trains of ultrashort optical pulses in the visible and near-infrared spectral region. In recent years, increasing interest has been shown in ultrashort laser pulses in the ultraviolet and infrared. Novel ultrafast optical modulation techniques developed and used for self-mode-locked Ti:Sapphire (Ti:S) lasers have allowed powerful femtosecond-pulse generation with unprecedented stability, reliability, and reproducibility. These advances have led to a revival of the interest in nonlinear optical processes for frequency conversion of ultrashort pulses. The concomitant appearance of highdamage-threshold synthetic nonlinear insulating crystals triggered the development of efficient optical parametric generators and oscillators, as well as harmonic generators, giving rise to a substantial extension of the spectral coverage of ultrashort-pulse sources in the ultraviolet and infrared. Last but not least, the advent of laboratory-scale high peak power amplifiers based on solid state or excimer gain media has opened the way towards the efficient generation of short-wavelength radiation for the characterization of ultrashort pulses has become an important issue. ${ }^{1-3}$ Commercial manufactures of lasers claim that their equipment can emit almost perfect pulses, although in everyday experience, that is not true. An almost perfect pulse means an almost Gaussian profile without chirp with $\Delta \tau \Delta \omega \approx 1 / 2$, where $\Delta \tau$ is the duration of the pulse and $\Delta \omega$ its spectral width.

Roughly, the optical pulse can be characterized by its spectral width $(\Delta \omega)$ and by its time duration $(\Delta \tau)$. The product $(\Delta \omega)(\Delta \tau)$ can be considered as a measure of the perfection of the pulse. We suppose that a perfect pulse has no internal structure, and its $(\Delta \omega)(\Delta \tau)$ product is as low as possible. This means, that the squared modulus of $f(t)$ and its Fourier-transform $g(\omega)$ have an almost-Gaussian profile, such that $(\Delta \omega)^{2}(\Delta \tau)^{2} \approx 1 / 4$ should be accomplished.

The characterization of an optical pulse by its intensity profile and intensity of its spectrum is also interesting due to the analogy with the reconstruction of the transversal structure of a monochromatic beam by its intensity detected in the image and Fourier planes. ${ }^{4,5}$

Send correspondance to:

R.O.M. : E-mail: roberto@aleph.cinstrum.unam.mx

C.J.R.M.: E-mail: crm@labvis.unam.mx 
Distortions of the perfect Gaussian pulse are properly described by Hermite-Gauss functions which are self-Fourier transformed, and which give information about the class of these distortions. ${ }^{6,7}$

Here, we will describe the class of phase distortions which are very difficult to detect using methods proposed in these papers.

The aim of this work is to consider the spectral properties of almost-Gaussian functions. We will apply them to the characterization to the second order approximation in the expansion of the coefficients of the almost-perfect optical pulses given by Ti:S laser. ${ }^{8}$

\section{LINEARIZED APPROACH}

Let us have some pulse $E(t)$. It's registration of the intensity profile $|E(t)|^{2}$ and the spectral intensity profile $|\tilde{E}(\omega)|^{2}$. Are these quantities enough to distinguish this pulse from another pulse $E_{0}(\mathrm{t})$, or not? Of course, the intensity is measured with some error; so, the difference can be characterized by the defined error-bar functions:

$$
\mathcal{U}=\int_{-\infty}^{\infty}\left(|E(t)|^{2}-\left|E_{0}(t)\right|^{2}\right)^{2} d t \quad \text { and } \quad \mathcal{W}=\int_{-\infty}^{\infty}\left(|\tilde{E}(\omega)|^{2}-\left|\tilde{E}_{0}(\omega)\right|^{2}\right)^{2} d \omega
$$

Both functions are. in principle, measurable. For ultra-short pulses, we usually have the autocorrelation in eq. (1) instead of the intensity. It has been shown that, from the modulus of the function and the modulus of its Fourier-transform. the function can be effectively reconstructed, ${ }^{4,5}, 9$ at least if the function has finite carriage. This means that a segment $t_{1} \leq t_{2}$ exist such that a function $E(t)=0$ if $t \leq t_{1}$ or $t \geq t_{2}$. So, we could expect that the difference between $E^{\prime}$ and $E_{0}$ could cause a significant increase of the error-bar functions $\mathcal{U}$ and $\mathcal{W}$.

To work with adimensional quantities, we make the transformations $t \rightarrow \frac{t-t_{0}}{\Delta \tau}$, and $\omega \rightarrow \frac{\omega-\omega_{0}}{\Delta \omega}$, where $t_{0}$ is the "mean" time. $\Delta \tau$ is the root mean square (rms) width of the pulse, and $\omega_{0}$ and $\Delta \omega$ its spectral bandwidth. Let us assume that both $E$ and $E_{0}$ are almost Gaussian, after this transformation, i. e., $E(t) \approx E_{0}(t) \approx e^{-\frac{t^{2}}{2}}$, then we may expand them by the Hermite-(iauss functions as

$$
\begin{aligned}
E(t) & =\sum_{n=0}^{\infty} C_{n} \tilde{H}_{n}(t) e^{-\frac{t^{2}}{2}} \\
E_{0}(t) & =\sum_{n=0}^{\infty} S_{n} \tilde{H}_{n}(t) e^{-\frac{t^{2}}{2}}
\end{aligned}
$$

where $\tilde{H}_{n}(t)=H_{n}(t) / \sqrt{2^{n} \sqrt{\pi} n !}$ and $H_{n}(t)$ are the Hermite polynomials. ${ }^{10}$

For the Hermite-(iauss functions, we have the following theorem:

$$
\mathcal{F}\left(H_{n}(t) e^{-\frac{t^{2}}{2}}, t ; \omega\right)=i^{n} H_{n}(\omega) e^{-\frac{\omega^{2}}{2}}
$$

This is clear, if we treat $H_{n}(t) e^{-\frac{t^{2}}{2}}$ as an eigenfunction of the hamiltonian of the quantum harmonic oscillator. ${ }^{9}$ A different proof of this theorem which uses only the properties of the Hermite polynomials and no quantum analogies is given by Ortega ot al.

Using this theorem. We can write the expansion for the Fourier-tranforms of functions $E$ and $E_{0}$ :

$$
\begin{aligned}
& \tilde{E}(\omega)=\sum_{n=0}^{\infty} i^{n} C_{n} \tilde{H}_{n}(\omega) e^{-\frac{\omega^{2}}{2}} \\
& \tilde{E}_{0}(\omega)=\sum_{n=0}^{\infty} i^{n} S_{n} \tilde{H}_{n}(\omega) e^{-\frac{\omega^{2}}{2}} .
\end{aligned}
$$


Note that here, coefficients $C_{n}$ and $S_{n}$ are the same as in (2) and (3). Without loss of generality, we may set $C_{0}=S_{0}=1$, then, we have

$$
\begin{aligned}
& |E(t)|^{2}=\left[1+\sum_{n=1}^{\infty}\left(C_{n}+C_{n}^{*}\right) \tilde{H}_{n}(t)+\sum_{n, l=1}^{\infty} C_{n} C_{l}^{*} \tilde{H}_{n}(t) \tilde{H}_{l}(t)\right] e^{-t^{2}} \\
& \left|E_{0}(t)\right|^{2}=\left[1+\sum_{n=1}^{\infty}\left(S_{n}+S_{n}^{*}\right) \tilde{H}_{n}(t)+\sum_{n, l=1}^{\infty} S_{n} S_{l}^{*} \tilde{H}_{n}(t) \tilde{H}_{l}(t)\right] e^{-t^{2}}
\end{aligned}
$$

Replacing eqs. (7) and (8) and their Fourier-transform in eq. (1), respectively, and after some algebraic manipulations, we get

$$
\mathcal{U}=\sum_{m=1}^{\infty} \sum_{n=1}^{\infty} \frac{4 \operatorname{Re}\left(\alpha_{n}\right) \operatorname{Re}\left(\alpha_{m}\right)}{\sqrt{2^{n+m} \pi m ! n !}}(-1)^{\frac{m-n}{2}} 2^{\frac{m+n-1}{2}} \Gamma\left(\frac{m+n+1}{2}\right)
$$

and in the frequency-domain

$$
\mathcal{W}=\sum_{m=1}^{\infty} \sum_{n=1}^{\infty} \frac{4 \operatorname{Re}\left(i^{n} \alpha_{n}\right) \operatorname{Re}\left(i^{m} \alpha_{m}\right)}{\sqrt{2^{n+m} \pi m ! n !}}(-1)^{\frac{m-n}{2}} 2^{\frac{m+n-1}{2}} \Gamma\left(\frac{m+n+1}{2}\right)
$$

The "deviation" of the pulse $E$ from $E_{0}$ can be characterized by $\mathcal{V}=\mathcal{U}+\mathcal{W}$.

In order to understand the meaning of eqs. (9) and (10) let's consider the special case, $\alpha_{n}=\varepsilon \delta_{n k}$, for some fixed $k$. Then, eqs (9) and (10) can be written, respectively, as

$$
u=\frac{4 \Gamma(k+1 / 2)}{\sqrt{2 \pi} k !}(\operatorname{Re}(\varepsilon))^{2}
$$

and

$$
\mathcal{W}=\frac{4 \Gamma(k+1 / 2)}{\sqrt{2 \pi} k !}\left(\operatorname{Re}\left(i^{k} \varepsilon\right)\right)^{2}
$$

Hence, for odd $k$ we have

$$
\begin{aligned}
\mathcal{V}=\mathcal{U}+\mathcal{W} & =\frac{4 \Gamma(k+1 / 2)}{\sqrt{2 \pi} k !}|\varepsilon|^{2} \\
& =\frac{4(2 k-1) ! !}{\sqrt{2} 2^{k} k !}|\varepsilon|^{2}
\end{aligned}
$$

and for even $k$,

$$
\begin{aligned}
\mathcal{V}=\mathcal{U}+\mathcal{W} & =\frac{8 \Gamma(k+1 / 2)}{\sqrt{2 \pi} k !}(\operatorname{Re}\{\varepsilon\})^{2} \\
& =\frac{8(2 k-1) ! !}{\sqrt{2} 2^{k} k !}(\operatorname{Re}\{\varepsilon\})^{2}
\end{aligned}
$$

In $\mathcal{V}$ for even $k$, we have $(\operatorname{Re}\{\varepsilon\})^{2}$, but not $|\varepsilon|^{2}$ as for odd $k$. So if we add the small imaginary amount $i e^{-\frac{t^{2}}{2}} H_{2 l}(t)$, to the Gaussian pulse, in the first approximation the error bar deviation function does not change!

For example at $k=3, \quad \mathcal{V}=\frac{5}{4 \sqrt{2}}\left|\alpha_{3}\right|^{2}$.

while for $k=4, \quad \mathcal{V}=\frac{35}{32 \sqrt{2}} 4\left[\operatorname{Re}\left\{\alpha_{4}\right\}\right]^{2}$.

Hence, distortions will be hard to define at the quadratic order of perturbation with respect to $\varepsilon$, at $k=2 l, \alpha_{n}$ $=i \varepsilon$. 
Generally, the distortion which, in the first approximation, does not change $\mathcal{U}+\mathcal{W}$, can be represented as a sum of Hermite-Gaussian functions of the form:

$$
\delta E(t)=\sum_{l=2}^{\infty} i \varepsilon_{l} \tilde{H}_{2 l}(t) e^{-\frac{t^{2}}{2}}
$$

where $\varepsilon_{l}$ are small amounts.

In the linearized approximation, $E(t)$ and $E(t)+\delta E(t)$ have the same square of the modulus and the same square of the Fourier-transform.

\section{NUMERICAL EXAMPLES}

In this section, we present some examples to illustrate the statement follows eqs. (13) and (14). In the adimensional coordinates, we simulate the characterization with quadratic detectors of the self-Fourier Gaussian $u_{0}(t)=$ $\exp \left(-t^{2} / 2\right)$. In the simplest case, we disturb this perfect (Gaussian $u_{0}$ with small amount of the normalized HermiteGaussian function, so the pulse $u$ has the form

$$
u(t)=\left(1+\alpha H_{n}(t)\right) \exp \left(-t^{2} / 2\right)
$$

Figures $1 \mathrm{a}$ and $2 \mathrm{~b}$ represents the square of modulus of $u$, while the right side shows the square of modulus of its Fourier-transform. We plot it at $\alpha=1 / 4$ for $n=3$ and $\alpha=i / 4$ for $n=4$. As the reference line, we repeat the case $\alpha=0$ (perfect Gaussian) at each plot. Figure (2a) is the same as figure (2b) as it should be for the self-Fourier function.

In the case of polynomial of 3-rd order, the distortion reveals in the modulus of function in the time-domain (Fig. 1a), but not in the frequency-domain (Fig. 1b) for a perturbation complex coefficient with null imaginary part. The opposite holds for a perturbation with cero real part.

As for the 4-th order polynomial, it gives the self-Fourier function. So, distortions are seen as in the modulus of the function, as in the modulus of its Fourier transform, while the distortion has non-zero real part (Fig. 2a). But if we add such distortion with small imaginary coefficient, as in figures $2 b$, the distortions are almost not seen. As we have showed, the same would take place for a distortion of form of any Hermite-Gaussian function of even order, or their linear combination.

Any even function can be expanded with Hermite-Gaussian functions of even order. If this function is real, small imaginary amount of such a function added to the perfect Gaussian, is not seen at the second-order characterization.

\section{EXPERIMENTAL PROCEDURE}

The source of ultrashort laser pulses is a Ti:Sapphire Mira 900 pumped by an Innova $310 \mathrm{Ar}^{3+}$ laser from Coherent. Pulses are time-measured by a Femtochrome FR103-XL autocorrelator which uses a second harmonic as nonlinearity and captures the nonlinear signal with a photomultiplier. The autocorrelation traces are displayed and recorded with the aided of a digital oscilloscope Tektronix model TDS 744A. The experimental setup can be seen in Fig. 3. Digital data are fitted by Hermite-Gauss series.

The incoming pulses at $\lambda=800 \mathrm{~nm}$ in the autocorrelator are splitted by a pellicle beam splitter (PBS) into two replicas, one of them travells a variable-delay line while the other goes throught a fixed-delay line. A focus mirror (CM) combine both replica on a KDP nonlinear crystal (NLC) producing a second harmonic signal of fundamental pulses $(\lambda=400 \mathrm{~nm})$. This signal is captured by a photomultiplier (PMT) tube and displayed on the digital oscilloscope. A rotating mirror stage (RS) supply the variable-delay line. A corner-cube retroreflector mirror (CCM) on a sliding stages gives the fixed-delay path; this sliding stage is manually controlled by micromicrometer knob. Fig. 4 is a schematics of the autocorrelator. 

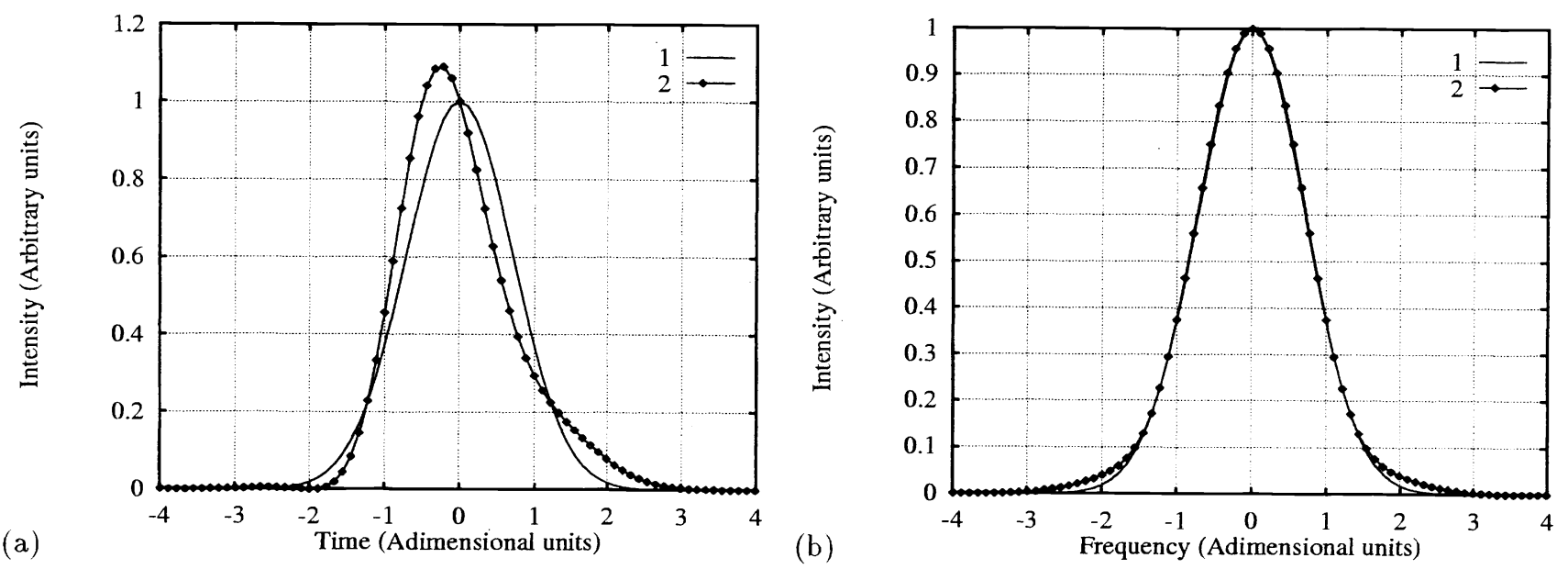

Figure 1. Intensity of a perfect-gaussian pulse (1). A small real 3rd order Hermite-Gauss perturbation has been added to the gaussian pulse (2). (a) Square modulus of $u_{0}(t)=e^{-t^{2}}$ and $u(t)=\left|1+\frac{1}{4} H_{3}(t)\right|^{2} e^{-t^{2}}$. (b) Square modulus of $\tilde{u}_{0}(\omega)=e^{-\omega^{2}}$ and $\tilde{u}(\omega)=\left|1-\frac{i}{4} H_{3}(\omega)\right|^{2} e^{-\omega^{2}}$.
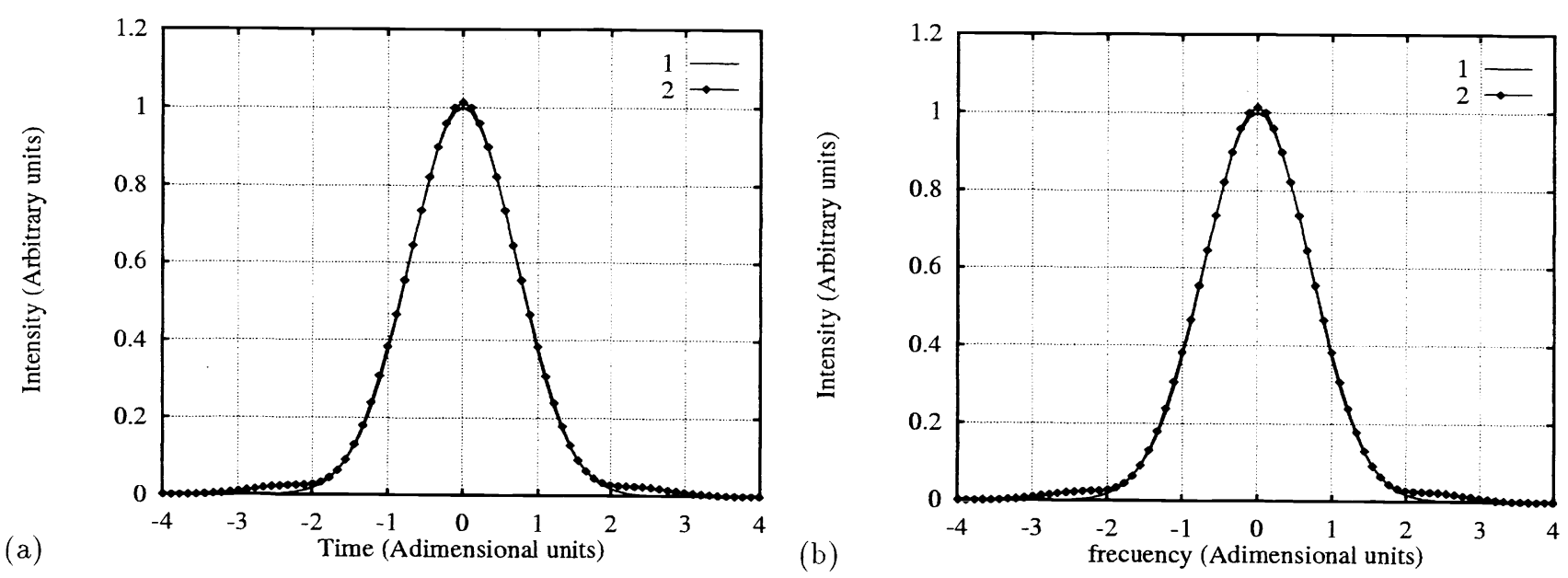

Figure 2. Intensity of a perfect-gaussian pulse (1). A small "pure" imaginary $4^{\text {th }}$ order Hermite-Gauss perturbation has been added to the gaussian pulse (2). (a) Square modulus of $u_{0}(t)=e^{-t^{2}}$ and $u(t)=\left|1+\frac{i}{4} H_{4}(t)\right|^{2} e^{-t^{2}}$. (b) Square modulus of $\tilde{u}_{0}(\omega)=e^{-\omega^{2}}$ and $\tilde{u}(\omega)=\left|1+\frac{i}{4} H_{4}(t)\right|^{2} e^{-\omega^{2}}$. Note the symmetry: Fig. (2b) is the same as Fig. (2a) because they are self-Fourier functions. 


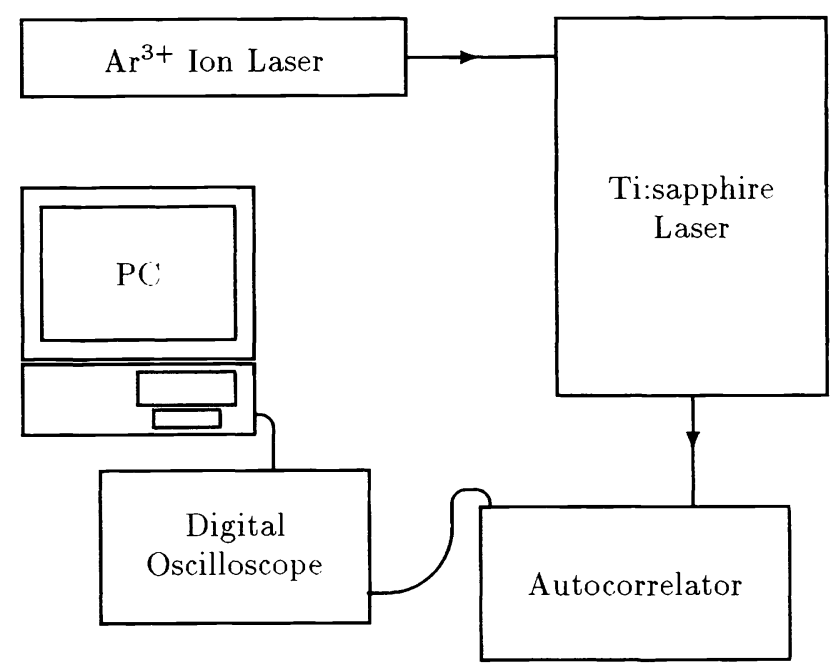

Figure 3. Femtosencod Laser Sistem at CIUNAM.

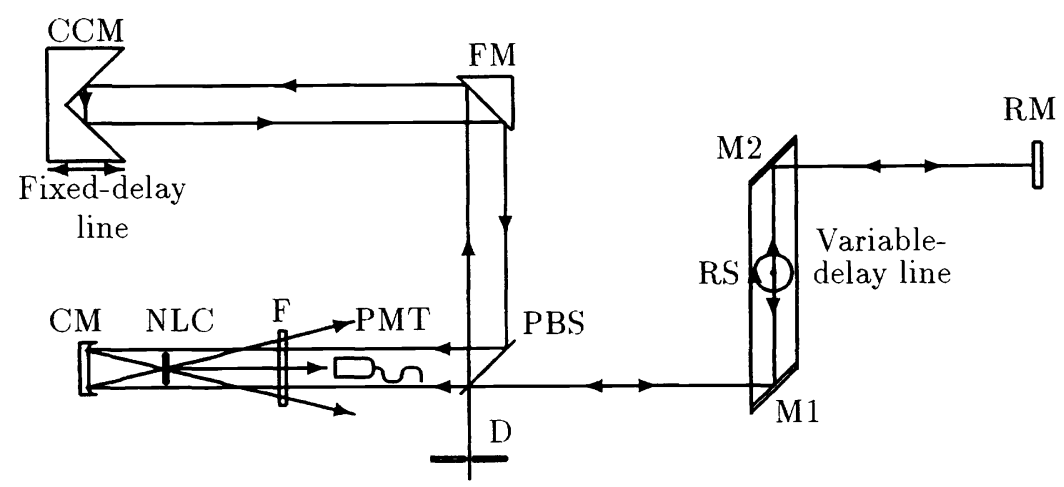

Figure 4. Schematics of the autocorrelator. 


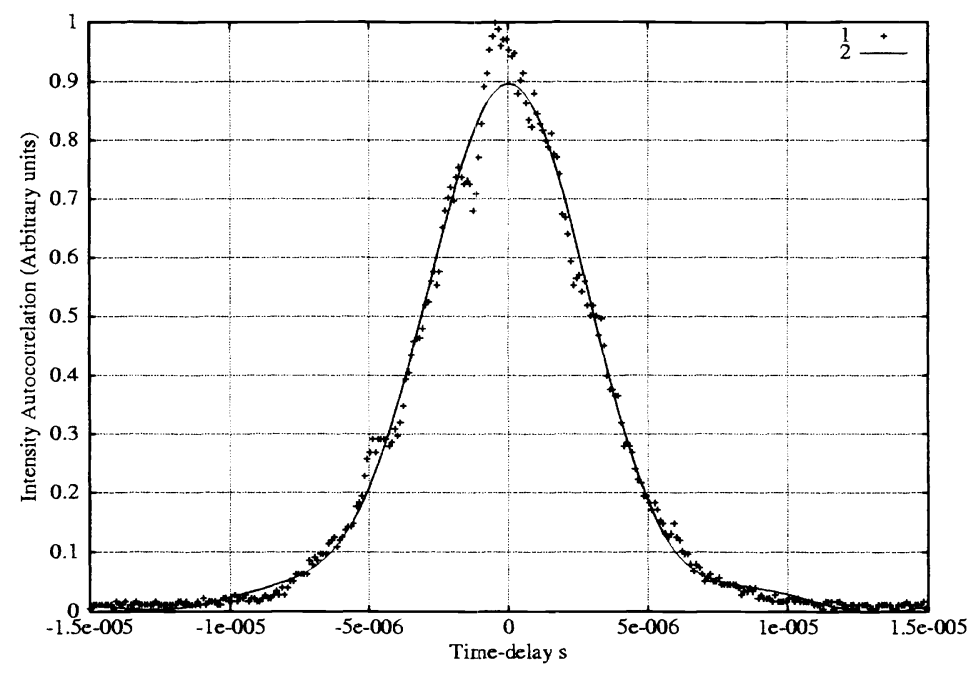

Figure 5. (1) Intensity autocorrelation experimental data, (2) $10^{\text {th }}$-order Hermite-Gauss summatory fitting.

\section{RESULTS}

As we said in Section 2, one usually measure the autocorrelation of $|E(t)|^{2}$ instead the intensity itself. Figure 5 shows an experimental autocorrelation trace $(+)$ sampled at 500 mega samples per second (MS/s) by a digital oscilloscope. These experimental data were fitted by a $10^{\text {th }}$-order Hermite-Gauss finite summatory that resembles Eq. (2), whose coefficients are $c_{0}=8.72537 \times 10^{-1}, c_{1}=-8.48329 \times 10^{-3}, c_{2}=-2.59739 \times 10^{-1}, c_{3}=1.08901 \times 10^{-3}, c_{4}=$ $1.35207 \times 10^{-1}, c_{5}=2.81173 \times 10^{-3}, c_{6}=-5.31421 \times 10^{-2}, c_{7}=-4.92186 \times 10^{-3}, c_{8}=4.76055 \times 10^{-2}, c_{9}=$ $6.36713 \times 10^{-3}, c_{10}=-1.59361 \times 10^{-2}$.

It is clear that $c_{0}$ is the dominant coefficient in this approximation. So the envelop of the trace is almost-Gaussian, hence the envelop of the pulse is also almost-Gaussian. If we take in considerations higher order coefficients, more time-components of pulses can be determined.

The rms width of the autocorrelator trace is $4.698 \mu \mathrm{s}$, then assuming a factor form of 0.707 for a Gaussian envelop, we got 108 fs as the rms width of the pulse.

\section{CONCLUSIONS}

The product of the duration of the pulse and its spectral width is considered as a measure of the perfection of the pulse. We found the specific class of distortions of an almost-Gaussian wave packet, in the quadratic approximation, which change nor the modulus of the function neither the modulus of its Fourier-tranform.

It would be interesting to consider some elementary processes which are sensitive to such distortions of a quasiGaussian pulse. Experimentally, to obtain the spectra of the pulse to get a complete characterization of the electric field of the pulse.

With this work we start a new line of research at our University, where students from chemistry, physics, and electrical engineering will be training in the new ultrafast laser techniques. Mathematical concepts, such as Fourier transform, cross and autocorrelation; characterization techiques, like FROG, time-frequency techniques and its vast applications in physical optics, dispersion, nonlinear optics, and ultrafast spectroscopy.

\section{AKNOWLEDGEMENTS}

We thank N. Bruce, D. Kouznetsov, and K. B. Wolf for their comments on the text. We also thank to Mrs. H. Estrada and R. Pérez for their help in preparation of the text. C. J. Roman had a support from DGAPA-UNAM (Project Num. 1N104597) and from CONACyT (Project \# 4480-A9406). R. Ortega bought the femtosystem with BID-UNAM \#95-B3-C-DGP-L0034-1077, also is a member of the Sistema Nacional de Investigadores (\#6095). 


\section{REFERENCES}

1. L. Sarger and J. Oberlé, How to measure the characteristics of laser pulses, ch. 7, pp. 177-201. Springer-Verlag, Germany, 1 ed., 1998.

2. T. D. Donnelly and C. Grossman, "Ultrafast fenomena: A laboratory experiment for undergraduates.," $A m$. J. Phys. 66, pp. 677-685, August 1998.

3. P. Loza-Alvarez, D. T. Reid, M. Ebrahimzadeh, W. Sibbett, H. Karlsson, P. Henriksson, G. Ardvidsson, and F. Laurel, "Periodically-polled $\mathrm{RbTiOAsO}_{4}$ femtosecond optical parametric oscillator tunable from 1.38 to $1.58 \mu \mathrm{m}, "$ Appl. Phys. B 68, pp. 177-180, 1999.

4. R. W. Gerchberg and W. O. Saxton, "A practical algorithm for the determination of phase from image and diffraction plane pictures," Optik 35(2), pp. 237-246, 1972.

5. T. I. Kuznetsova and D. Y. Kuznetsov, "A phase retrieval algorithm based on analogy for optical image processing," Optics Comm. 61, pp. 374-377, March 1987.

6. R. Ortega-Martínez, C. J. Román-Moreno., and D. Y. Kouznetsov, "On the second order characterization of ultrashort laser pulses. (Accepted for publication in)," Journal of Modern Optics , March 1999.

7. C. J. Román-Moreno, "Determinación de la amplitud y de la fase de un pulso láser ultracorto usando funciones de Hermite-Gauss." M. Sc. thesis, Facultad de Ciencias, U.N.A.M., México, D. F., February 1999.

8. C. J. Román-Moreno and R. Ortega-Martínez, "Caracterización temporal de los pulsos ultracortos de un láser de Titanio:Zafiro con un autocorrelador," in SOMI XII. Congreso de Instrumentación., pp. 579-583, Sociedad Mexicana de Instrumentación, A. C., (San Luis Potosí, S, L, P.), October 1997.

9. L. D. Landau and E. M. Lifshitz, Quantum Mechanics (Non-Relativistic Theory), vol. 3 of Course of Theoretical Physics, Pergamon Press. 3rd ed., 1977.

10. M. Abramowitz and I. A. Stegun, Handbook of Mathematical Functions, Dover Publications, 1972. 